\title{
Fouille de forums de discussion en santé : Identification des caractéristiques des contributeurs
}

\author{
Thierry Hamon ${ }^{1,4}$, Camille Pertin ${ }^{1}$, Carole Deccache ${ }^{2,3}$, \\ Rémi Gagnayre ${ }^{2}$
}

\author{
1. LIMSI, CNRS, Université Paris-Saclay, Orsay, France \\ \{camille.pertin,thierry.hamon\}@limsi.fr \\ 2. Université Paris 13, LEPS, Sorbonne Paris Cité, Bobigny, France \\ \{carole.deccache,remi.gagnayre\}@univ-paris13.fr \\ 3. Clinique du Château de Verhnes, Bondigoux, France
}

4. Université Paris 13, Sorbonne Paris Cité, Villetaneuse, France

\begin{abstract}
RÉSUMÉ. Les forums de discussion en santé sont des sources précieuses pour l'éducation thérapeutique. Il est ainsi possible d'étudier le comportement des contributeurs mais aussi leur compréhension de la maladie et leurs besoins face à leurs problèmes de santé. Cependant, l'analyse des échanges et des comportements nécessite de disposer d'informations démographiques sur les intervenants. Nous présentons une approche combinant des lexiques et des ressources terminologiques à des classifieurs supervisés pour identifier la tranche d'âge, le genre des utilisateurs, leur maladie et la relation entre l'auteur du message et le patient. Notre approche est testée sur des forums de discussion en français liés au diabète. Les résultats obtenus sont variables suivant les informations recherchées. L'identification du genre et de la tranche d'âge est de bonne qualité tandis que l'extraction de la maladie ou du statut du contributeur est plus difficile mais encourageante.

ABSTRACT. Health online discussion fora are precious sources of information from the therapeutic education. They permit to study the behaviours of the participants but also their understanding of their disorder and their needs when facing health problems. Yet, the analysis of the exchanges between users and their behaviour requires to collect demographic information on the internet users posting messages. We present an approach combining lexica and terminological resources with supervised classifiers for the identification of age and gender of users, their disorder and relation between the user and the patient. We apply our approch on diabetes mellitus discussion fora in French. Results vary according to the extracted information. Gender and age range detection achieves a good quality while disorders and relation between the author of the message and the patient are more difficult to identify.
\end{abstract}

Technique et science informatique $-\mathrm{n}^{\circ} 1-6 / 2018,105-125$ 
MOTS-CLÉS : Traitement Automatique des Langues, Forum de discussion, Education thérapeutique, Informations démographiques, Relation patient-auteur

KEYWORDS: Natural Language Processing, Online discussion forum, Therapeutic education, Demographic information, Patient-author relation

DOI:10.3166/TSI.37-0005 @ 2018 Lavoisier

\section{Introduction}

Depuis plusieurs années maintenant, Internet est devenu la source principale d'informations pour les patients en quête de connaissances sur leurs maladies et leurs traitements (Murray et al., 2003 ; Wald et al., 2007). Alors que de nombreux documents médicaux (articles scientifiques, articles journalistiques, etc.) sont facilement accessibles et peuvent aider les patients à comprendre et gérer leurs maladies (Diaz et al., 2002 ; McMullan, 2006), les patients internautes utilisent également beaucoup les média sociaux, et en particulier les forums de discussion dédiés à la santé (Seybert, Reinecke, 2013), pour y trouver d'autres informations de santé et des témoignages. Les forums de discussion apparaissent ainsi comme un des premiers outil d'échange d'informations employé par les utilisateurs d'Internet pour la santé (Price et al., 2014 ; Communication, Patient\&Web, 2013 ; Ramsay, 2016). Il s'agit de lieux virtuels d'échange d'informations consacrés au thème de la santé ou de la maladie. Les internautes, malades ou non-malades, y interagissent en posant des questions ou en y répondant.

Ce constat est d'autant plus vrai que, pour les patients atteints de maladies chroniques comme le diabète, ou pour leur entourage, les forums de discussion liés à la santé sont des lieux importants pour l'expression et le partage de l'expérience de leur vie quotidienne (Harry, 2011 ; Yasri-Labrique, 2011 ; Lederman et al., 2014; Smedley et al., 2015). Une enquête européenne a montré que les forums de discussion en santé sont utilisés par $19 \%$ des personnes asthmatiques interrogées et sont le deuxième outil après les moteurs de recherche pour chercher de l'information (Price et al., 2014). En France, un malade chronique sur deux utilise les forums de discussion en santé (Communication, Patient\&Web, 2013).

Ces communautés en ligne sont également reconnues aujourd'hui commme des sources précieuses d'informations dans le cadre de l'éducation thérapeutique du patient (Harry, 2011). Les échanges entre patients ou leur entourage permettent ainsi d'étudier leur compréhension de la maladie, leur comportement et leurs besoins face à leurs problèmes de santé, ainsi que les stratégies d'apprentissage qu'ils mettent en œuvre. Nous nous intéressons ici à l'analyse d'échanges entre les internautes diabétiques francophones sur des forums de discussion dédiés.

Ces analyses du comportement des patients internautes nécessitent de disposer d'informations sur leur profil, et notamment d'informations démographiques comme le genre ou la tranche d'âge, mais aussi l'indication de la maladie à laquelle est 
confrontée l'internaute (Baumann et al., 2017 ; Choi et al., 2017 ; Li et al., 2017). Or ces informations sont rarement disponibles, surtout explicitement, sur les forums de discussion (voir section 3), le pseudo-anonymat étant une caractéristique prépondérante des forums de santé (Scola-Streckenbach, 2008). Il est donc nécessaire de proposer des approches automatiques pour identifier les informations démographiques qui nous intéressent. En outre, nous nous intéressons à la relation entre l'auteur du message et le patient ${ }^{1}$. De plus, pour mieux cerner les comportements des internautes diabétiques, nous souhaitons également reconnaitre le type de diabète dont la personne est atteinte.

Dans la suite de ce travail, nous présentons tout d'abord un état de l'art sur les approches identifiant les informations démographiques (section 2). À la section 3, nous introduisons les forums de discussion dédiés au diabète et les ressources utilisés, et nous présentons une première analyse manuelle montrant les difficultés de la tâche. Nous décrivons alors notre approche pour identifier automatiquement les informations ciblées (section 4). Après avoir présenté et discuté les résultats (section 6), nous concluons et proposons quelques perspectives.

\section{Travaux existants}

\subsection{Identification des problèmes médicaux}

L'extraction et la catégorisation d'informations sont des tâches typiquement réalisées sur les données textuelles du domaine médical. Ainsi, la reconnaissance de pathologies est une tâche classique (Cohen, Hunter, 2008 ; Rzhetsky et al., 2009). Énormément de travaux s'y sont intéressés en particulier pour identifier dans des textes cliniques les maladies dont souffrent les patients (Chen et al., 2008 ; Halgrim et al., 2010) et pour extraire de la littérature scientifique des évènements médicaux ou biologiques mais aussi des relations entre des maladies et des médicaments (Ananiadou et al., 2010; Miwa et al., 2012; Xu, Wang, 2013). Les approches s'appuient généralement sur des terminologies médicales comme le MeSH (MeSH, 1998) ou la Snomed CT (IHTSDO, 2008) et peuvent éprouver des difficultés à identifier les noms de maladies lorsque les textes sont bruités ou la qualité rédactionnelle est faible comme c'est le cas avec les documents cliniques.

Les forums de discussion sont généralement exploités pour extraire des informations à partir des messages, comme les effets indésirables de médicaments (Stanovsky et al., 2017 ; Morlane-Hondère et al., 2016) mais aussi les mésusages (Bigeard et al., 2018). Les approches doivent faire face à plusieurs problèmes. Outre le caractère particulièrement bruité des textes traités, qui est un frein important à la reconnaissance de noms de maladies, elles doivent également faire face aux différences de dénominations qui existent entre le langage expert utilisé par les professionnels de santé, et présent dans les terminologies médicales, et le langage non-expert utilisé par les contributeurs des forums, la plupart du temps non expert (Antoine, Grabar, 2017).

$\overline{\text { 1. L'internaute auteur }}$ du message est-il le patient ou quelqu'un qui lui est proche? 
Afin de contourner ces problèmes, une solution consiste à faire émerger des lexiques spécifiques qui combinent des lexiques existants et des méthodes de clustering comme word2vec (Mikolov et al., 2013) ou la LDA (Blei et al., 2003) comme proposé dans les travaux existants (Bigeard, 2017; Nzali et al., 2017; Skeppstedt et al., 2018).

\subsection{Identification des informations démographiques}

Les messages publiés sur les forums de discussion et de manière générale sur les réseaux sociaux sont également exploités pour caractériser les contributeurs avec des informations démographiques (genre, âge), dans différentes perspectives : sociologique (Dickerson et al., 2004 ; Fisher et al., 2006 ; Akrich, Méadel, 2009 ; Kharrazi, 2009 ; Yu et al., 2012), de pharmacovigilance (Bigeard et al., 2018), d'éducation thérapeutique (Doumont, Aujoulat, 2002 ; Thoër, Aumond, 2011 ; Glasgow et al., 2012; Li et al., 2017) ou de santé publique (Benamara et al., 2018). Dans de nombreux cas, l'identification des informations démographiques est une étape préliminaire à une analyse manuelle des messages, étant donné les difficultés liées à une analyse automatique comme évoqué ci-dessus.

La reconnaissance des catégories démographiques (genre, âge) a suscité de nombreux travaux dans le cadre du profilage d'auteurs sur Twitter (Rangel et al., 2015) et de la détection de plagiat (Barrón-Cedeño et al., 2010). Les approches proposées pour identifier le genre dans les média sociaux, que ce soit des forums de discussion, Twitter ou Facebook, utilisent des méthodes de classification supervisées, comme des SVM et des arbres de décision (Burger et al., 2011 ; Zamal et al., 2012 ; Liu, Ruths, 2013 ; Santosh et al., 2013 ; Estruch et al., 2017). Une grande variété de descripteurs est utilisée : date de publication, émoticônes, images, pseudonyme, prénom et nom de l'intervenant, nombre de followers, racines de mots, n-grams de caractères, de mots ou de catégories morpho-syntaxiques. La précision obtenue varie entre $56 \%$ et $87 \%$. Aussi, l'exploitation de descripteurs très simples (n-grammes de caractères extraits du nom des utilisateurs) montre une précision allant jusqu'à $89 \%$ (Littlestone, 1988). Les participants à la compétition $\mathrm{PAN}^{2}$ atteignent des résultats similaires sur la détection du genre des internautes à partir de tweets (Pardo et al., 2017). Pour détecter l'âge des contributeurs de réseaux sociaux, quelques travaux ont également exploité les méthodes supervisées comme des SVM (Zamal et al., 2012) ou des réseaux bayésiens (Mechti et al., 2016) mais aussi des approches semi-supervisées (Ardehaly, Culotta, 2015). Les descripteurs utilisés sont généralement les mêmes que ceux retenus pour l'identification du genre. Les précisions obtenues varient entre $60 \%$ et $80 \%$.

\subsection{Identification du rôle des intervenants}

Les travaux s'intéressant à caractériser le rôle de contributeurs de forums de discussion visent principalement à différencier trois catégories d'intervenants : les pa-

2. https://pan.webis.de/index.htm 
tients, les professionnels de santé en général, et les experts ou les spécialistes (Abdaoui et al., 2014 ; Opitz et al., 2014 ; Lu et al., 2017 ; Rajabi, Abidi, 2017). Nous n'avons pas trouvé de travaux qui cherchent à calculer la relation entre l'auteur du message et le patient. Les approches proposées exploitent des informations décrivant les messages (nombre de phrases par message, nombre de messages, etc.) ainsi que leur contenu (fréquence du vocabulaire, présence de termes issus de terminologies comme le MeSH, etc.). Suivant les objectifs, ces descripteurs sont ensuite utilisés par (i) des méthodes d'apprentissage supervisé pour classer les intervenants par catégorie (Abdaoui et al., 2014), (ii) des mesures de centralité pour analyser les relations entre les contributeurs et pour identifier les professionnels de santé les plus influents et les participants clés (Rajabi, Abidi, 2017), ou (iii) des méthode de clustering non supervisé pour faire émerger les groupes de contributeurs (Lu et al., 2017).

\section{Matériel}

Nous présentons les forums de discussion exploités, une analyse manuelle des messages d'un des forums afin d'appréhender la difficulté d'identification des informations démographiques, et les ressources exploitées.

\subsection{Description et analyse des forums de discussion sur le diabète}

Plusieurs travaux ont montré que l'absence de soignants dans les forums facilite le partage d'expériences entre les internautes et fournit ainsi des contenus propres aux ressentis des patients (Harry et al., 2008 ; Medina et al., 2013). Nous avons donc sélectionné deux forums francophones dédiés au diabète : LesDiabétiques ${ }^{3}$ et FemmesDiabétiques ${ }^{4}$. Le premier forum, créé en juin 2004, réunit des internautes majoritairement canadiens mais aussi français ou plus généralement francophones. Les messages sont classés dans 19 thématiques. Le second forum, créé en octobre 2006, offre son soutien aux diabétiques et à leurs proches. Les discussions sont regroupées en 45 thématiques traitant d'aspects variés de la vie des patients. Tout comme le forum précédent, il est indépendant des professionnels de la santé. Étant donné que ce forum s'adresse en premier lieu à un public féminin, on y trouve de nombreuses discussions en lien avec les particularités de grossesses chez les patientes diabétiques, par exemple. Tout en étant beaucoup moins nombreux, les utilisateurs masculins y interviennent également. Bien que, selon la loi lors de la collecte des données et les recommandations de la CNIL, aucune autorisation n'est nécessaire pour recueillir des informations publiques diffusées sur le web, nous avons mis en place avec l'aide de conseillers de la CNIL, une procédure de recueil des données a été mise en place permettant de respecter la législation et de protéger les internautes. Cette procédure consiste à entrer en contact avec le webmaster des forums pour obtenir son accord et pour que ce dernier diffuse un message d'information sur le forum. Nous

3. http://www.lesdiabetiques.com/forum

4. http://femmesdiabetiques.com/forum 
avons réalisé une récupération des forums à des fins d'analyse. Afin de protéger les personnes, toutes les informations utilisent aux traitements automatiques (présence de prénom, de date de naissance, etc. ont été représentés sous forme de booléens et de valeurs numériques lorsque nous souhaitions utiliser le nombre d'occurrence. Toutes les données recueillies sont stockées dans un fichier déclaré auprès de la CNIL conformément à la législation française (Déclaration $\mathrm{n}^{\circ}$ 1752627) et sécurisé par un mot de passe. De plus, nous avons obtenu l'accord préalable du conseil d'administration du forum FemmesDiabétiques. Avant la phase d'extraction automatique des données, une lettre d'information a été postée sur le forum, dans un fil de discussion dédié à la recherche et également diffusée dans la newsletter du forum. L'accord d'un Comité de Protection des Personnes a été obtenu.

Au cours du premier semestre 2017, nous avons récupéré l'ensemble des fils de discussion de ces deux forums depuis leur création. Le tableau 1 présente le nombre de fils de discussion et de messages, le nombre d'internautes et le nombre d'occurrences de mots. La figure 1 présente la distribution du nombre de messages par internaute sur chacun des deux forums. Les informations démographiques, et plus généralement les métadonnées associées aux internautes, sont disparates et très incomplètes. Par exemple, si le genre est disponible sur les deux forums, moins de $15 \%$ d'internautes le renseignent (tableau 2). Il en est de même pour les tranches d'âge : seuls $38 \%$ d'internautes de Femmes Diabétiques mentionnent leur date de naissance, rendant ainsi possible l'identification de leur tranche d'âge (tableau 3).

Forum LesDiabetiques

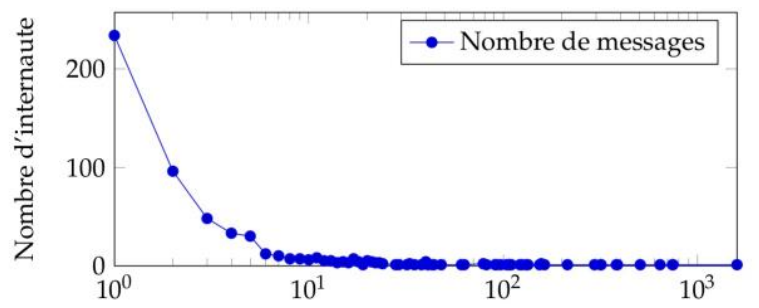

Forum FemmesDiabetiques

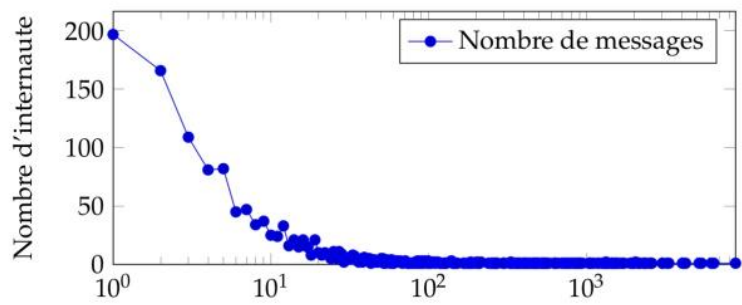

Figure 1. Distribution du nombre de messages par internaute

Notons que les signatures des internautes peuvent également fournir des informations utiles, comme par exemple Maman x 3, Papa de..., l'âge, le type et la durée du 
Tableau 1. Description des forums de discussion

\begin{tabular}{c|c|c|c|c} 
& \multicolumn{4}{|c}{ Nombre de } \\
Forum & fils disc. & messages & internautes & mots \\
\hline LesDiabétiques & 1563 & 11040 & 586 & 943358 \\
FemmesDiabétiques & 4770 & 186246 & 1400 & 10782312
\end{tabular}

Tableau 2. Répartition du genre des internautes l'ayant explicitement indiqué

\begin{tabular}{c|c|c|c|c} 
Forum & $\begin{array}{c}\text { Nombre } \\
\text { d'internautes }\end{array}$ & Mention du genre & Féminin & Masculin \\
\hline LesDiabétiques & 586 & $48(8 \%)$ & 26 & 22 \\
FemmesDiabétiques & 1400 & $222(16 \%)$ & 217 & 5
\end{tabular}

diabète (Diabète de type 1 depuis 21 ans) ou même le protocole ou le matériel utilisé (pompe Animas, Traîtement : 1 Lantus + 3 NovoRapid/jour). Cependant, celles-ci ne sont disponibles que sur le forum LesDiabétiques. De plus, la signature associée aux messages est celle au moment de la lecture des messages et non celle au moment où le message a été posté. Elle peut donc contenir des informations à jour aussi bien que des informations obsolètes. Il est alors possible qu'elles soient décorrélées du message au moment de sa publication. Leur utilisation peut s'avérer difficile.

\subsection{Annotation manuelle}

Les observations ci-dessus montrent qu'il est nécessaire de disposer de méthodes automatiques pour une identification plus exhaustive d'informations sur les patients : genre, âge, pathologie et relation entre l'auteur du message et le patient. Ainsi, deux annotateurs ont effectué l'annotation du genre et de la tranche d'âge des internautes de LesDiabétiques. Ces annotations servent de référence pour le développement et l'évaluation de méthodes automatiques, et permettent de définir la difficulté de la tâche. Ayant à disposition le pseudonyme de l'internaute, le sujet des discussions, les

Tableau 3. Répartition de la tranche d'âge des internautes ayant explicitement indiqué leur âge

\begin{tabular}{c|c}
\multicolumn{2}{c}{ Forum Femmes Diabétiques } \\
Tranche d'âge & Nombre d'internautes \\
\hline bébé (B) / 0-3 & 0 \\
enfant (E) / 4-12 & 1 \\
adolescent (a) / 13-18 & 0 \\
jeune adulte (J) / 19-25 & 24 \\
adulte actif (A) / 26-60 & 506 \\
senior (s) / 61-80 & 5 \\
senior+ (S) / 81-150 & 0 \\
\hline Total & 536
\end{tabular}


112 TSI. Volume $37-\mathrm{n}^{\circ} 1-6 / 2018$

Tableau 4. Annotation manuelle de référence du forum LesDiabétiquesTranches d'âge des internautes.

\begin{tabular}{c|c} 
Tranche d'âge & Nombre d'internautes \\
\hline bébé (B) / 0-3 & 0 \\
enfant (E) / 4-12 & 0 \\
adolescent (a) / 13-18 & 15 \\
jeune adulte (J) / 19-25 & 57 \\
adulte actif (A) / 26-60 & 199 \\
senior (s) / 61-80 & 13 \\
senior+ (S) / 81-150 & 0 \\
inconnu & 249 \\
études & 53 \\
\hline Total & 586
\end{tabular}

Tableau 5. Annotation manuelle de référence du forum LesDiabétiques-Genre des internautes.

\begin{tabular}{c|c} 
Genre & nombre \\
\hline $\mathrm{M}$ & 141 \\
$\mathrm{~F}$ & 289 \\
inconnu & 103 \\
études & 53 \\
\hline Total & 586
\end{tabular}

messages postés et la signature d'un utilisateur, les annotateurs avaient pour tâche de lui attribuer son genre (tableau 5) et sa tranche d'âge (tableau 4).

Nous avons également observé que le forum comporte plusieurs messages correspondant à des études pour lesquelles les informations démographiques ne sont pas pertinentes. Ces messages ont été identifiés spécifiquement afin de ne pas les prendre en compte par la suite.

Afin d'estimer l'accord entre les annotateurs et la difficulté des deux tâches d'annotation, nous avons calculé le Kappa de Cohen sur les annotations :

$$
\kappa=\frac{\underline{P}_{a}-P_{e}}{1-P_{e}}
$$

où $P_{a}$ est la proportion d'annotations identiques observées et $P_{e}$ est la proportion théorique d'annotations identiques mais obtenues aléatoirement. Celui-ci indique un accord inter-annotateurs fort $(0,67)$ pour le genre et modéré $(0,562)$ pour la tranche d'âge. La phase de consensus a également montré que les problèmes d'identification du genre étaient principalement liés au volume important de texte à lire et à une surinterprétation de certains indices. Par exemple, alors qu'il n'existe pas d'indices explicites, la lecture des messages peut donner l'impression qu'il est possible d'attribuer un genre à l'internaute. Par ailleurs, il apparaît que le début des premiers messages d'un 
internaute est crucial pour retrouver les informations recherchées. En dehors de l'indication explicite du genre, les prénoms sont également des indices importants, tout comme les accords d'adjectifs (prêt/prête). L'identification de la tranche d'âge est plus complexe : l'âge peut être indiqué dans la signature ou dans le premier message, lorsque l'internaute se présente, mais dans de nombreux cas, un calcul supplémentaire est nécessaire, comme dans : je suis diabétique depuiis l'âge de 16 ans c'est à dire depuis 3 ans et demi.

En revanche, l'identification des pathologies et de la relation entre l'auteur du message et le patient, n'a pu être réalisée que par un seul annotateur. Les tableaux 6 et 7 présentent la distribution des annotations pour ces deux types d'information. Les données s'avèrent également déséquilibrées : les pathologies concernent principalement les diabètes de type 1 et 2 (77\%), et les rédacteurs des messages sont en grande majorité les patients $(73,7 \%)$. De même que précédemment, dans nos expériences, nous avons écarté les messages des contributeurs dont il n'était pas possible de déterminer la catégorie (inconnu).

Tableau 6. Annotation manuelle de référence du forum LesDiabétiquesPathologie (DID : Diabète Insulino-dépendant, DT1 : Diabète de type 1, DT2 : Diabète de type 2, DT : Diabète)

\begin{tabular}{c|c|c|c} 
Type de maladie & Nombre & \multicolumn{2}{c}{} \\
\hline DID & 1 & DT2 sous insuline & 13 \\
DT1 ou DT2 & 109 & DT Gestationel & 2 \\
DT1 & 219 & DT réactif & 1 \\
DT1 et DT2 & 2 & Pied de charcot & 1 \\
DT2 & 122 & Prédiabétique & 2 \\
DT2 +insuline & 1 & suspicion DT & 13 \\
inconnu & 100 & & \\
\hline \multicolumn{2}{c|}{ Total } & & 586
\end{tabular}

Tableau 7. Annotation manuelle de référence du forum LesDiabét iquesRelation patient-rédactur ( $I$ : l'internaute est le patient).

\begin{tabular}{c|c|c|c} 
Lien de parenté & Nombre & Lien de parenté & Nombre \\
\hline I & 432 & Enfant & 2 \\
I et Mère & 1 & Fille & 6 \\
Mère & 17 & Sour & 3 \\
Parent & 4 & Tante & 1 \\
Père & 10 & Ami & 3 \\
Conjoint & 18 & inconnu & 89 \\
\hline \multicolumn{2}{c|}{ Total } & \multicolumn{2}{|c}{586}
\end{tabular}

\subsection{Ressources}

Pour identifier le genre à partir du pseudonyme, nous avons constitué une ressource de 3206 prénoms à partir de plusieurs sources disponibles sur Wikipédia. Les 92 pré- 
noms ambigus (Dominique, Camille) y sont marqués comme tels explicitement. Cette ressource a été nettoyée pour ne conserver que le prénom, ses éventuelles variantes et son genre ou son ambiguïté.

Les premières observations réalisées sur le corpus nous ont conduits à faire l'hypothèse que les flexions audibles au féminin (comme dans prête ou curieuse) seraient de bons indicateurs pour identifier le genre des internautes. Ainsi, nous avons créé une ressource à partir de représentations phonologiques des adjectifs du lexique lexique. org ${ }^{5}: 5163$ adjectifs, qui possèdent plusieurs représentations phonologiques dont une avec la flexion au féminin, ont été sélectionnés.

Nous avons également constitué un lexique de 24 mots pouvant être des indices du genre des internautes comme maman, grossesse, mari, épouse, etc.

\section{Méthodes}

Nous proposons d'utiliser plusieurs algorithmes de classification supervisée disponibles dans Weka : J48, REPTree, RandomTree, RandomForest, BayesNet, LibSVM avec un noyau linéaire et MultiBoostAB avec un bayésien naïf comme classifieur faible. Les arbres de décision obtenus avec J48 et REPTree peuvent être visualisés pour mieux analyser le modèle construit et les descripteurs exploités. L'unité traitée est l'internaute, auquel nous associons tous ses messages postés dans un forum donné. Suivant les catégories, les expériences réalisées s'appuient sur différents ensembles de descripteurs. A partir d'expériences préliminaires, nous avons établi les meilleures combinaisons utilisant les informations suivantes :

- genre : prénoms, adjectifs avec l'accord au féminin audible dans le texte et les signatures lorsque disponibles, âge du contributeur, lexique des mots marqueurs du genre, texte des messages et des signatures. On a donc les combinaisons suivantes pour

- le forum LesDiabétiques : tous les descripteurs sans les adjectifs avec l'accord au féminin audible $\left(C_{1}\right)$, tous les descripteurs sans l'âge du contributeur ni les mots marqueurs du genre $\left(C_{2}\right)$, tous les descripteurs sans l'âge du contributeur $\left(C_{3}\right)$;

- le forum FemmesDiabétiques : tous les descripteurs sans l'âge $\left(C_{1}\right)$, tous les descripteurs sans le texte $\left(C_{2}\right)$, tous les descripteurs $\left(C_{3}\right)$.

- tranche d'âge : prénoms, suppression de valeurs numériques dans les pseudonymes, reconnaissance de prénoms dans le pseudonyme, texte des messages et des signatures, 3 premières et la dernière mentions d'années dans les messages, genre de l'internaute. On a donc les combinaisons suivantes pour les deux forums : tous les descripteurs sans le genre $\left(C_{1}\right)$, tous les descripteurs sans le genre et le vocabulaire du texte $\left(C_{2}\right)$, tous les descripteurs sans le vocabulaire du texte et de la signature $\left(C_{3}\right)$

- pathologie : nombre et longueur moyenne de sujets et de messages, présence d'une signature et de prénoms dans la signature, textes des signatures, message et 
sujet, 3 premières et la dernière mentions d'années dans les messages. Tous les descripteurs ont été utilisés lors de nos expériences.

- relation patient-auteur : nombre et longueur moyenne de sujets et de messages, présence d'une signature et de prénoms dans la signature, le vocabulaire issu du texte des signatures, message et sujet, 3 premières et la dernière mentions d'années dans les messages, âge du contributeur, lexique des mots marqueurs du genre. On a donc les combinaisons suivantes pour le forum LesDiabétiques : tous les descripteurs sans l'âge du contributeur ni les mots marqueurs du genre $\left(\left(C_{1}\right)\right.$, tous les descripteurs sans l'âge du contributeur ni le vocabulaire issu du texte des signatures, message et sujet $\left(C_{2}\right)$, tous les descripteurs sans le vocabulaire issu du texte des signatures, message et sujet $\left(C_{3}\right)$.

Les catégories visées dépendent également des expériences :

- genre : 2 catégories (M, F);

- tranche d'âge : 7 catégories, comme dans le tableau 3;

- relation patient-auteur : 6 catégories (ami, conjoint, enfant, internaute lui-même, oncle-tante, parent);

- pathologie : 12 catégories selon les types de diabète et les co-morbidités fréquentes.

\section{Expériences et évaluation}

Nous avons réalisé deux types d'expériences :

- un modèle appris sur un forum est testé sur les données du même forum, et dans ce cas nous effectuons une validation croisée à 10 plis;

- le modèle appris sur le forum LesDiabétiques, qui a des données de référence plus complètes, et ensuite testé sur le forum FemmesDiabétiques. Ceci permet de tester la généricité des modèles et des informations à extraire.

Les résultats ont été évalués à l'aide des mesures de micro et de macro-précision (Sebastiani, 2002). La micro-précision (équation 1) fournit des indications sur la qualité des résultats au niveau de chaque pseudonyme, tandis que la macro-précision (équation 2) décrit le comportement du système indépendamment de la taille des classes. TP correspond aux vrais positifs, c'est-à-dire les réponses correctes fournies par le systèmes, et FP correspond aux faux positifs, c'est-à-dire les réponses incorrectes. Étant donné le déséquilibre des données, nous avons également pris en compte la diversité des classes prédites dans notre évaluation.

$$
\text { précision } \sum_{\mu}=\sum_{i=1}^{\sum_{n}\left(\mathrm{TP}_{i}+\mathrm{FP}_{i}\right)} \quad \text { (1) précision }=\frac{\sum_{n=1}-_{i \mathrm{TP}_{i}} \Sigma}{n}
$$


Sur le corpus LesDiabétiques nous avons utilisé les annotations réalisées comme références, c'est-à-dire le genre, la tranche d'âge, la pathologie et la relation entre le patient et l'auteur du message. Sur le corpus FemmesDiabétiques, les références sont les informations partielles dont nous disposons, c'est-à-dire le genre pour 222 pseudonymes (16\%), la tranche d'âge pour 536 pseudonymes (38\%) mais essentiellement dans la même catégorie (adulte), et le type de diabète pour 581 pseudonymes $(41,5 \%)$ avec seulement 4 catégories (diabète type 1 et diabète type 2 , diabète et diabète gestationnel).

\section{Résultats et discussion}

Nous effectuons donc quatre séries d'expériences pour détecter les informations relatives au genre du patient, à sa tranche d'âge, à sa pathologie et à la relation entre le patient et l'auteur du message, grâce aux annotations manuelles et aux métadonnées. Comme décrit à la section 4, plusieurs combinaisons de descripteurs ont été testées avec différents algorithmes. Nous ne présentons que les résultats les plus intéressants.

Les tableaux 8 et 9 présentent les résultats obtenus pour la détection du genre sur chaque forum de discussion. Ces résultats sont conformes à l'état de l'art et aux résultats obtenus par les participants aux campagnes PAN (Pardo et al., 2017) bien que le type de textes soit différent. En particulier, malgré le déséquilibre entre les catégories du genre sur le forum FemmesDiabétiques, l'utilisation de l'ensemble des descripteurs et notamment de la tranche d'âge $\left(C_{1}\right.$ vs. $\left.C_{3}\right)$, permet d'obtenir de bons résultats avec les classifieurs LibSVM et J48. En revanche, la combinaison $C_{2}$ s'avère inexploitable puisque les deux classifieurs précédents regroupent l'ensemble des contributeurs dans la classe prépondérante $(\mathrm{f})$. Nous pouvons faire l'hypothèse que les contributeurs de ce forum semblent avoir un style rédactionnel ou un comportement qui varie en fonction de l'âge.

Bien que la combinaison $C_{1}$ avec $\mathrm{J} 48$ n'exploite pas les adjectifs dont l'accord au féminin est audible (tableau 10), nous avons pu observer sur l'ensemble des expériences réalisées que ceux-ci jouent un rôle important en particulier avec un réseau bayésien (BayesNet). L'analyse des arbres de décision confirme cette observation. Cependant, le nombre d'adjectifs réellement utilisés est très limité comparé à la liste totale d'adjectifs disponibles. Les adjectifs les plus utiles sont essentiellement des adjectifs très communs, comme contente, inquiète, haute, heureuse ou des adjectifs relatifs au fonctionnement de forums, comme inscrite. À part un adjectif (normale), tous les adjectifs utilisés ont une flexion en genre audible, ce qui semble indiquer que ce sont effectivement de bons indicateurs du genre des personnes. Dans ce genre d'écrits, la phonétisation de l'écriture (avec la flexion féminine audible) semble réduire les fautes d'orthographe et aider en effet la reconnaissance du genre. De plus, l'utilisation des mots marqueurs du genre tend à permettre d'améliorer la macro-précision.

L'identification de la tranche d'âge est une tâche plus complexe (tableau 10). Quelles que soient les combinaisons utilisées, la macro-précision est assez faible (de l'ordre de $30 \%$ ). Les différentes catégories sont difficiles à discriminer et aucun descripteur utilisé ne semblent avoir un impact réel sur les résultats. L'utilisation ou non 
Tableau 8. Résultats de la détection du genre sur le forum LesDiabétiques $\left(C_{1}\right.$ : tous les descripteurs sans les adjectifs avec l'accord au féminin audible, $C_{2}$ : tous les descripteurs sans l'âge du contributeur ni les mots marqueurs du genre, $C_{3}$ : tous les descripteurs sans l'âge du contributeur).

\begin{tabular}{l|l|lll} 
& & BayesNet & LibSVM & $\mathrm{J} 48$ \\
\hline$C_{1}$ & prec $_{M}$ & 68,41 & 74,18 & 78,94 \\
& prec $_{\mu}$ & 68,09 & 74,47 & 78,72 \\
& classes & $\mathrm{f}, \mathrm{m}$ & $\mathrm{f}, \mathrm{m}$ & $\mathrm{f}, \mathrm{m}$ \\
\hline$C_{2}$ & prec $_{M}$ & 73,95 & 70,84 & 71,51 \\
& prec $_{\mu}$ & 78,19 & 74,25 & 75,64 \\
& classes & $\mathrm{f}, \mathrm{m}$ & $\mathrm{m}, \mathrm{f}$ & $\mathrm{m}, \mathrm{f}$ \\
\hline$C_{3}$ & prec $_{M}$ & 74,01 & 72,45 & 72,57 \\
& prec $_{\mu}$ & 76,80 & 74,94 & 76,57 \\
& classes & $\mathrm{f}, \mathrm{m}$ & $\mathrm{f}, \mathrm{m}$ & $\mathrm{f}, \mathrm{m}$
\end{tabular}

Tableau 9. Résultats de la détection du genre sur le forum FemmesDiabétiques $\left(C_{1}\right.$ : tous les descripteurs sans l'âge, $C_{2}$ : tous les descripteurs sans le texte, $C_{3}$ : tous les descripteurs).

\begin{tabular}{l|l|lll} 
& & BayesNet & LibSVM & $\mathrm{J} 48$ \\
\hline$C_{1}$ & prec & 66,48 & 74,18 & 80,86 \\
& prec $_{\mu}$ & 65,96 & 74,47 & 80,85 \\
& classes & $\mathrm{f}, \mathrm{m}$ & $\mathrm{m}, \mathrm{f}$ & $\mathrm{m}, \mathrm{f}$ \\
\hline$C_{2}$ & prec $_{M}$ & 69,77 & 49,77 & 50,00 \\
& prec $_{\mu}$ & 98,20 & 97,30 & 97,75 \\
& classes & $\mathrm{f}, \mathrm{m}$ & $\mathrm{f}$ & $\mathrm{f}$ \\
\hline$C_{3}$ & prec $_{M}$ & 59,80 & 83,24 & 82,78 \\
& prec $_{\mu}$ & 59,57 & 82,98 & 82,98 \\
& classes & $\mathrm{m}, \mathrm{f}$ & $\mathrm{f}, \mathrm{m}$ & $\mathrm{f}, \mathrm{m}$
\end{tabular}

de l'ensemble du vocabulaire des messages et des signatures dégradent assez peu les performances quel que soit l'algorithme de classification. Bien que les classes soient très déséquilibrées sur le forum FemmesDiabétiques, les chiffres obtenus indiquent qu'il existe une bonne concordance entre ce que déclarent les internautes et ce qui peut être calculé par les méthodes supervisées et ce grâce à l'analyse du texte des messages.

Dans le tableau 11, nous pouvons voir que la détection de pathologies présente également beaucoup de difficultés, avec des micro-précisions de l'ordre de $60 \%$ sur LesDiabétiques et $80 \%$ sur Femmes Diabétiques mais des macro-précisions d'environ 15 et $8 \%$ respectivement. Nous présentons ici la combinaison de descripteurs et d'algorithmes permettant d'obtenir plusieurs classes. En effet, dans les autres cas, les algorithmes de classification ont privilégié la classe prépondérante (DT1 pour diabète de type 1) comme par exemple l'algorithme REPTree appliqué aux données du forum FemmesDiabétiques. Par ailleurs, dans des expériences préliminaires, 
118 TSI. Volume $37-n^{\circ} 1-6 / 2018$

Tableau 10. Résultats de la détection des tranches d'âge sur les deux forums $\left(C_{1}\right.$ : tous les descripteurs sans le genre, $C_{2}$ : tous les descripteurs sans le genre et le vocabulaire du texte, $C_{3}$ : tous les descripteurs sans le vocabulaire du texte et de la signature).

\begin{tabular}{l|l|lll|lll} 
& & \multicolumn{3}{|c|}{ LesDiabétiques } & \multicolumn{3}{c}{ FemmesDiabétiques } \\
& & $\mathrm{J} 48$ & BayesNet & REPTree & $\mathrm{J} 48$ & BayesNet & REPTree \\
\hline$C_{1}$ & prec $_{M}$ & 33,00 & 30,56 & 28,74 & 14,29 & 14,26 & 14,29 \\
& prec $_{\mu}$ & 81,34 & 78,87 & 78,52 & 94,40 & 94,22 & 94,40 \\
& classes & $\mathrm{A}, \mathrm{s}, \mathrm{a}, \mathrm{J}$ & $\mathrm{J}, \mathrm{A}, \mathrm{s}$ & $\mathrm{J}, \mathrm{A}, \mathrm{s}$ & $\mathrm{A}$ & $\mathrm{A}$ & $\mathrm{A}$ \\
\hline$C_{2}$ & prec $_{M}$ & 32,93 & 30,52 & 29,62 & 14,14 & 14,21 & 14,21 \\
& prec $_{\mu}$ & 80,99 & 79,58 & 78,52 & 69,37 & 69,72 & 69,72 \\
& classes & $\mathrm{J}, \mathrm{A}, \mathrm{a}, \mathrm{s}$ & $\mathrm{J}, \mathrm{s}, \mathrm{A}$ & $\mathrm{J}, \mathrm{a}, \mathrm{s}, \mathrm{A}$ & $\mathrm{A}$ & $\mathrm{A}$ & $\mathrm{A}$ \\
\hline$C_{3}$ & prec & 33,63 & 30,45 & 28,60 & 14,29 & 14,21 & 14,29 \\
& prec $_{\mu}$ & 80,99 & 79,23 & 77,82 & 70,07 & 69,72 & 70,07 \\
& classes & $\mathrm{J}, \mathrm{A}, \mathrm{s}, \mathrm{a}$ & $\mathrm{A}, \mathrm{s}, \mathrm{J}$ & $\mathrm{J}, \mathrm{A}, \mathrm{s}$ & $\mathrm{A}$ & $\mathrm{J}, \mathrm{A}$ & $\mathrm{A}$
\end{tabular}

nous avions cherché à exploiter des termes français issus de l'UMLS. Les résultats obtenus n'étaient pas non plus concluants: le vocabulaire et les « termes » utilisés par les internautes diffèrent souvent de ceux présents dans les terminologies ou employés par les experts du domaine. Un moyen de contourner cet écueil serait d'utiliser des méthodes de détection de paraphrases ou de gloses (Grabar, Hamon, 2015).

Tableau 11. Résultats de l'identification de la pathologie sur les deux forums (utilisation de tous les descripteurs)

\begin{tabular}{l|ll|lll} 
& \multicolumn{2}{|c|}{ LesDiabétiques } & \multicolumn{3}{c}{ FemmesDiabétiques } \\
& LibSVM & REPTree & LibSVM & RandomForest & REPTree \\
\hline prec $_{M}$ & 14,68 & 15,94 & 8,26 & 8,45 & 8,33 \\
prec $_{\mu}$ & 60,20 & 61,69 & 83,15 & 78,28 & 83,90 \\
classes & DT1,DT,DT2 & DT1,DT,DT2 & DT1 & DT,DT2,DT1 & DT1
\end{tabular}

L'identification de la relation patient-auteur sur le forum LesDiabétiques est également complexe mais donne des résultats encourageants (tableau 12). Nous présentons ici les résultats permettant de discriminer les classes, à l'exception de J48 avec la série de descripteurs $C_{1}$. Ainsi, nous pouvons observer que le classifieur bayésien naïf et l'extension de l'algorithme AdaBoost MultiBoostAB offrent des performances intéressantes bien qu'assez faibles. Les combinaisons de descripteurs utilisées semblent indiquer que le genre de l'auteur du message ( $C_{1}$ vs. $C_{2}$ et $\left.C_{3}\right)$ mais aussi des informations temporelles $\left(C_{1}\right.$ et $C_{2}$ vs. $\left.C_{3}\right)$ sont des informations importantes dans ce contexte.

Le tableau 13 présente les résultats de l'application du modèle généré à partir du forum LesDiabétiques sur le forum FemmesDiabétiques. Nous avons utilisé les combinaisons de descripteurs et les classifieurs montrant de meilleures performances sur le forum LesDiabétiques (tableaux 8, 10 et 11). La comparaison 
Tableau 12. Résultats de l'identification de la relation patient-auteur sur le forum LesDiabétiques ( $C_{1}$ : tous les descripteurs sans l'âge du contributeur ni les mots marqueurs du genre, $C_{2}$ : tous les descripteurs sans l'âge du contributeur ni le vocabulaire issu du texte des signatures, message et sujet, $C_{3}$ : tous les descripteurs sans le vocabulaire issu du texte des signatures, message et sujet.

\begin{tabular}{|c|c|c|c|c|}
\hline & & MultiBoostAB (NB) & Naive Bayes & $\mathrm{J} 48$ \\
\hline \multirow[t]{4}{*}{$C_{1}$} & prec $_{M}$ & 4,83 & 6,41 & 5,88 \\
\hline & prec $_{\mu}$ & 24,29 & 19,80 & 87,55 \\
\hline & classes & Conjoint,I, & Fille,Mère,I, & I \\
\hline & & Mère,Fille & Père,Conjoint & \\
\hline \multirow[t]{4}{*}{$C_{2}$} & prec $M$ & 10,90 & 11,41 & 7,15 \\
\hline & prec $_{\mu}$ & 18,16 & 16,53 & 87,76 \\
\hline & classes & I,Mère,Ami,Père, & Conjoint,Mère,I, & Conjoint,I \\
\hline & & Parent,Conjoint & Fille,Père,Ami & \\
\hline \multirow[t]{4}{*}{$C_{2}$} & prec $_{M}$ & 10,95 & 9,83 & 7,11 \\
\hline & prec $_{\mu}$ & 18,98 & 16,94 & 87,14 \\
\hline & classes & Parent,Conjoint,Ami, & Fille,Mère,I, & I,Conjoint \\
\hline & & Père,I,Mère & Père,Ami,Conjoint & \\
\hline
\end{tabular}

Tableau 13. Résultats obtenus sur le forum FemmesDiabétiques avec le modèle appris sur le forum LesDiabétiques

\begin{tabular}{l|llll} 
Genre & & BayesNet & LibSVM & J48 \\
\hline$C_{2}$ & prec $_{M}$ & 75.39 & 83.09 & 75.39 \\
& prec $_{\mu}$ & 90.09 & 86.04 & 90.09 \\
& classes & m,f & m,f & f,m \\
\hline Age & & J48 & BayesNet & REPTree \\
\hline$C_{3}$ & prec $_{M}$ & 14.29 & 13.81 & 14.29 \\
& prec $_{\mu}$ & 94.40 & 87.50 & 94.40 \\
& classes & A & A,J & A \\
\hline Pathologie & & LibSVM & RandomForest & REPTree \\
\hline & prec $_{M}$ & 8.21 & 8,54 & 8.33 \\
& prec $_{\mu}$ & 73.41 & 73,78 & 83.90 \\
& classes & DT2,DT1,DT & DT1,DT & DT1
\end{tabular}

avec les résultats obtenus directement sur le forum Femmes Diabétiques montrent que l'identification du genre est équivalente sinon meilleure avec une macro-précision supérieure à $75 \%$ et une micro-précision variant entre 86 et $90 \%$. Les résultats de la détection des tranches d'âge sont également encourageants avec une macro-précision identique mais une meilleure micro-précision. Cependant, les modèles ont tendance à n'attribuer qu'une seule catégorie. En ce qui concerne l'identification des pathologies, les résultats sont légèrement dégradés ou équivalents. Les performances obtenues montrent ainsi qu'il est possible d'apprendre un modèle sur un forum pour lequel on 
dispose d'informations relatives à une catégorie et l'appliquer ensuite à un autre forum dans le même domaine.

\section{Conclusion et perspectives}

Au travers des expériences présentées, nous avons pu constater que les méthodes proposées (apprentissage supervisé, ressources et analyse du texte) permettent d'identifier certaines caractéristiques des utilisateurs des forums de santé. Nos résultats indiquent que certaines informations, en particulier le genre mais aussi la tranche d'âge sont plus aisées à détecter que d'autres (relation à l'auteur du message et la pathologie). Cependant, ce travail permet déjà de faire un premier pas vers la prédiction du profil des utilisateurs dans les forums de discussion. Ces informations fournissent les premières informations sur le type de la population que l'on retrouve sur ces forums. Elles sont utiles pour différents travaux de recherche, y compris l'analyse du parcours de santé et l'éducation thérapeutique, car dans les deux cas il est nécessaire de prendre en compte le vécu des patients.

Dans les travaux futurs, nous allons enrichir les données de référence pour effectuer d'autres expériences, avec une attention particulière pour la transposition de modèles de langue d'un forum à l'autre. Afin d'améliorer la reconnaissance des différences d'informations, d'autres descripteurs sont également envisagés comme des n-grams de mots ou de catégories morpho-syntaxiques. La pathologie pourrait également être considérée individuellement dans chaque message. Nous souhaitons étudier cette piste, l'identification pouvant alors être vue comme une analyse thématique de messages. Par ailleurs, les indicateurs acquis pourront être utilisés dans d'autres travaux de recherche, de même que fournir des indices aux professionnels du secteur médical et pour l'évolution des programmes d'éducation thérapeutique grâce à une meilleure caractérisation et compréhension de certains patients.

\section{Remerciements}

Cette recherche a bénéficié de l'aide des partenaires financeurs de l'IReSP dans le cadre de l'appel à projets général 2016 volet Services de Santé (projet GAGNAYREAAP16-HSR-6).

\section{Bibliographie}

Abdaoui A., Azé J., Bringay S., Grabar N., Poncelet P. (2014, octobre). Predicting Medical Roles in Online Health Fora. In SLSP: Statistical Language and Speech Processing, vol. LNCS, p. 247-258. Grenoble, France. Consulté sur https://hal-lirmm.ccsd.cnrs.fr/lirmm $-01130749$

Akrich M., Méadel C. (2009, octobre). Les échanges entre patients sur l'internet. La Presse Médicale, vol. 38, nº 10, p. 1484-1490.

Ananiadou S., Pyysalo S., Tsujii J., Kell D. B. (2010). Event extraction for systems biology by text mining the literature. Trends in Biotechnology, vol. 28, n 7 , p. 381-390. 
Antoine E., Grabar N. (2017). Acquisition of expert/non-expert vocabulary from reformulations. In Proceedings of mie 2017, vol. 235, p. 521-525.

Ardehaly E. M., Culotta A. (2015). Inferring latent attributes of twitter users with label regularization. In Proceedings of the 2015 conference of the north american chapter of the association for computational linguistics: Human language technologies, p. 185-195. Association for Computational Linguistics.

Barrón-Cedeño A., Rosso P., Agirre E., Labaka G. (2010, August). Plagiarism detection across distant language pairs. In Proceedings of the 23rd international conference on computational linguistics (coling 2010), p. 37-45. Beijing, China, Coling 2010 Organizing Committee.

Baumann E., Czerwinski F., Reifegerste D. (2017). Gender-specific determinants and patterns of online health information seeking: Results from a representative german health survey. Journal of Medical Internet Research, vol. 19, n ${ }^{\circ}$.

Benamara F., Moriceau V., Mothe J., Ramiandrisoa F., He Z. (2018). Automatic detection of depressive users in social media. In Actes de coria 2018.

Bigeard E. (2017). Construction de lexiques pour l'extraction des mentions de maladies dans les forums de santé. In Actes des 19eme rencontres jeunes chercheurs en informatique pour le tal (recital), p. 15-27.

Bigeard Élise, Grabar N., Thiessard F. (2018). Typology of drug misuse created from information available in health fora. In Proceedings of mie2018, vol. 247, p. 351-355.

Blei D. M., Ng A. Y., Jordan M. I. (2003). Latent dirichlet allocation. Journal of Machine Learning Research, p. 003-1022.

Burger J., Henderson J., Zarrella G. (2011). Discriminating gender on twitter. In Conference on empirical methods in natural language processing, p. 1301-1309.

Chen E., Hripcsak G., Xu H., Markatou M., Friedman C. (2008). Automated acquisition of disease drug knowledge from biomedical and clinical documents: an initial study. $\mathrm{J} \mathrm{Am}$ Med Inform Assoc, vol. 15, no 1, p. 87-98.

Choi M.-J., Kimd S.-H., Lee S., Kwon B. C., Yi J. S., Choo J. (2017). Toward predicting social support needs in online health social networks. Journal of Medical Internet Research, vol. $19, \mathrm{n}^{\circ} 8$, p.e 272 .

Cohen K. B., Hunter L. (2008). Getting started in text mining. PLoS Comput Biol, vol. 4, no 1 , p. e20.

Communication L., Patient\&Web. (2013). A la recherche du ePatient: Les français et l'internet santé. maladie chronique et numérique attitude. Paris, France.

Diaz J. A., Griffith R. A., Ng J. J., Reinert S. E., Friedmann P. D., Moulton A. W. (2002, March). Patients' use of the internet for medical information. J Gen Intern Med, vol. 17, no 3 , p. 180-185.

Dickerson S., Reinhart A. M., Feeley T. H., Bidani R., Rich E., Garg V. K. et al. (2004). Patient internet use for health information at three urban primary care clinics. JAMIA, vol. $11, \mathrm{n}^{\circ} 6$, p. $499-504$.

Doumont D., Aujoulat I. (2002). L'empowerment et l'éducation du patient (vol. 1). Bruxelles, UCL - RESO. (24 p.) 
122 TSI. Volume $37-\mathrm{n}^{\circ} 1-6 / 2018$

Estruch C. P., Palacios R. P., Paolo R. (2017). Learning multimodal gender profile using neural networks. In Proceedings of the international conference recent advances in natural language processing, ranlp 2017, p. 577-582. INCOMA Ltd.

Fisher D., Smith M., Welser H. T. (2006). You are who you talk to: Detecting roles in usenet newsgroups. In Proceedings of the 39th hawaii international conference on system sciences.

Glasgow R. E., Kurz D., King D., Dickman J. M., Faber A. J., Halterman E. et al. (2012). Twelve-month outcomes of an internet-based diabetes self-management support program. Patient Education and Counseling, vol. 87, no 1, p. 81-92.

Grabar N., Hamon T. (2015). Extraction automatique de paraphrases grand public pour les termes médicaux. In Actes de taln 2015, p. 182-193.

Halgrim S., Xia F., Solti I., Cadag E., Uzuner Özlem. (2010, June). Extracting medication information from discharge summaries. In Proceedings of the naacl hlt 2010 second louhi workshop on text and data mining of health documents, p. 61-67. Los Angeles, California, USA, Association for Computational Linguistics.

Harry I. (2011). Analyse des échanges écrits entre patients adultes et parents d'enfants, diabétiques de type 1, sur les forums d'internet : implication pour l'éducation thérapeutique du patient. Thèse de doctorat non publiée, Université Paris 13.

Harry I., Gagnayre R., d'Ivernois J.-F. (2008). Analyse des échanges écrits entre patients diabétiques sur les forums de discussion. Distances et savoirs, vol. 6, nº 3 .

IHTSDO. (2008). SNOMED Clinical Terms - Technical Reference Guide. International Health Terminology Standards Development Organisation. (http://www.ihtsdo.org/snomed-ct)

Kharrazi H. (2009). Improving healthy behaviors in type 1 diabetic patients by interactive frameworks. In Proceedings of amia 2009 symposium, p. 322-326.

Lederman R., Fan H., Smith S., Chang S. (2014). Who can you trust? credibility assessment in online health forums. Health Policy and Technology, vol. 3, p. 13-25.

Li X., Gray K., Verspoor K., Barnett S. (2017). Understanding the context of learning in an online social network for health professionals' informal learning. In Proceedings of mie 2017, vol. 235, p. 353-357.

Littlestone N. (1988). Learning quickly when irrelevant attributes abound: A new linearthreshold algorithm. Machine Learning, vol. 2, p. 285-318.

Liu W., Ruths D. (2013). What's in a name? Using first names as features for gender inference in twitter. In Aaai spring symposium, p. 10-16.

Lu Y., Wu Y., Liu J., Li J., Zhang P. (2017). Understanding health care social media use from different stakeholder perspectives: A content analysis of an online health community. Journal of Medical Internet Research, vol. 19, $\mathrm{n}^{\circ} 4$.

McMullan M. (2006, Oct). Patients using the internet to obtain health information: how this affects the patient-health professional relationship. Patient Education and Counselling, vol. $63, \mathrm{n}^{\circ} 1-2$, p. $24-28$.

Mechti S., Jaoua M., Faiz R., Bouhamed H., Belguith L. H. (2016). Author profiling: Age prediction based on advanced bayesian networks. In Proceedings of cicling 2016. 
Medina E. L., Filho O. L., Mesquita C. T. (2013). Health social networks as online life support groups for patients with cardiovascular diseases. Arq Bras Cardiol, vol. 101, n 2, p. e39e45.

MeSH. (1998). Medical subject headings. Library of Medicine, Bethesda, Maryland, WWW page http://www.nlm.nih.gov/mesh/meshhome.html,.

Mikolov T., Yih W.-t., Zweig G. (2013, June). Linguistic regularities in continuous space word representations. In Proceedings of the 2013 conference of the north american chapter of the association for computational linguistics: Human language technologies, p. 746-751. Atlanta, Georgia, Association for Computational Linguistics.

Miwa M., Thompson P., Ananiadou S. (2012). Boosting automatic event extraction from the literature using domain adaptation and coreference resolution. Bioinformatics, vol. 28, $\mathrm{n}^{\circ} 13$, p. $1759-65$.

Morlane-Hondère F., Grouin C., Zweigenbaum P. (2016). Identification of adverse drug reactions from social media. In Proc of lrec. Portorož, Slovenia.

Murray E., Lo B., Pollack L., Donelan K., Catania J., White M. et al. (2003, Jul). The impact of health information on the internet on the physician-patient relationship: patient perceptions. Arch Intern Med, vol. 163, n 14, p. 1727-1734.

Nzali M. D. T., Bringay S., Lavergne C., Mollevi C., Opitz T. (2017). What patients can tell us: Topic analysis for social media on breast cancer. JMIR Medical Informatics, vol. 5, nº 3 .

Opitz T., Aze J., Bringay S., Joutard C., Lavergne C., Mollevi C. (2014). Breast cancer and quality of life: Medical information extraction from health forums. In Proceedings of mie, vol. 205, p. 1070-1074.

Pardo F. M. R., Rosso P., Potthast M., Stein B. (2017). Overview of the 5th author profiling task at PAN 2017: Gender and language variety identification in twitter. In Working notes of CLEF 2017 - conference and labs of the evaluation forum, dublin, ireland, september 11-14, 2017. Consulté sur http://ceur-ws.org/Vol-1866/invited_paper_11.pdf

Price D., Fletcher M., Molen T. van der. (2014). Asthma control and management in 8,000 european patients: the recognise asthma and link to symptoms and experience (realise) survey. NPJ Primary Care Respiratory Medicine, vol. 24, $\mathrm{n}^{\circ} 14009$.

Rajabi E., Abidi S. S. R. (2017). Discovering central practitioners in a medical discussion forum using semantic web analytics. In Proceedings of mie 2017, vol. 235, p. 486-490.

Ramsay S. O. W. pour. (2016). Réseaux sociaux santé dans la prévention et l'éducation thérapeutique des français. Paris, France. Consulté sur http://publik-s.com/wp-content/uploads/ 2016/12/ETUDE-OPINIONWAY2016.pdf

Rangel F., Potthast M., Celli F., Stein B., Rosso P., Daelemans W. (2015, September). Overview of the 3rd author profiling task at pan 2015. In L. Cappellato, N. Ferro, G. Jones, E. S. Juan (Eds.), Clef 2015 labs and workshops, notebook papers. Toulouse, France.

Rzhetsky A., Seringhaus M., Gerstein M. B. (2009). Getting started in text mining: part two. PLoS Comput Biol, vol. 5, no 7, p. e1000411.

Santosh K., Bansal R., Shekhar M., Varma V. (2013). Author profiling: Predicting age and gender from blogs - notebook for pan at clef. In Proceedings of clef 2013. 
124 TSI. Volume $37-n^{\circ} 1-6 / 2018$

Scola-Streckenbach S. (2008). Experience-based information: The role of web-based patient networks in consumer health information services. $J$ Consum Health Internet, vol. 12, $\mathrm{n}^{\circ} 3$ p. 216-236.

Sebastiani F. (2002). Machine learning in automated text categorization. ACM Computing Surveys, vol. 34, no 1, p. 1-47.

Seybert H., Reinecke P. (2013). Three quarters of europeans used the internet in 2013. Eurostat, Statistics on focus.

Skeppstedt M., Kerren A., Stede M. (2018). Vaccine hesitancy in discussion forums: Computerassisted argument mining with topic models. In Proceedings of mie2018, vol. 247, p. 366370.

Smedley R., Coulson N., Gavin J., Rodham K., Watts L. (2015). Online social support for complex regional pain syndrome: A content analysis of support exchanges within a newly launched discussion forum. Computers in Human Behavior, vol. 51, p. 53-63.

Stanovsky G., Gruhl D., Mendes P. (2017). Recognizing mentions of adverse drug reaction in social media using knowledge-infused recurrent models. In Proceedings of the 15th conference of the european chapter of the association for computational linguistics: Volume 1, long papers, p. 142-151. Association for Computational Linguistics. Consulté sur http:// aclweb.org/anthology/E17-1014

Thoër C., Aumond S. (2011). Construction des savoirs et du risque relatifs aux médicaments détournés dans les forums sur internet [Building of the knowledge and the risk related to the drugs on the Internet fora]. Anthropologie et sociétés - Numéro spécial "Cyberespace et Anthropologie : transmission des savoirs et des savoir-faire", vol. 35, n 1-2.

Wald H. S., Dube C. E., Anthony D. C. (2007, Nov). Untangling the web. the impact of internet use on health care and the physician-patient relationship. Patient Education and Counselling, vol. $68, \mathrm{n}^{\circ} 3$, p. 218-224.

Xu R., Wang Q. (2013). Large-scale extraction of accurate drug-disease treatment pairs from biomedical literature for drug repurposing. BMC Bioinformatics.

Yasri-Labrique E. (2011). Les forums de discussion : agoras du XXIe siècle ? Paris, France, L'harmattan.

Yu C., Parsons J., Mamdani M., Lebovic G., Shah B., Bhattacharyya O. et al. (2012). Designing and evaluating a web-based selfmanagement site for patients with type 2 diabetes - systematic website development and study protocol. BMC Medical Informatics and Decision Making, vol. 12, n 1 , p. 57.

Zamal F., Liu W., Ruths D. (2012). Homophily and latent attribute inference: inferring latent attributes of Twitter users from neighbors. In International conference on weblogs and social media, p. 1-4.

Thierry Hamon. Thierry Hamon est Maître de conférences en informatique à l'Université Paris 13 et chercheur au LIMSI (UPR CNRS 3251). Ses travaux de recherche portent sur la mise au point de méthodes de Traitement Automatique des Langues pour l'acquisition de ressources terminologiques. Il s'intéresse également à l'utilisation de ces ressources dans divers cadres applicatifs comme la fouille de textes de spécialité, la recherche d'information 
ou la simplification de textes. Il est actuellement impliqué plusieurs projets ANR. Il a mis en oeuvre ses résultats de recherche dans plusieurs outils disponibles publiquement.

Camille Pertin. Camille Pertin était anciennement stagiaire au sein du LIMSI (UPR CNRS 3251) dans le cadre d'un diplôme de master en Traitement Automatique des Langues après avoir plus particulièrement étudié la linguistique. Son travail a consisté à rechercher des éléments langagiers permettant d'établir le profil des utilisateurs d'un forum de santé. Elle est actuellement enseignante dans le premier degré.

Carole Deccache. Carole Deccache est psychologue clinicienne et spécialiste en éducation thérapeutique du patient. Elle réalise son doctorat au Laboratoire Educations et Pratiques de Santé EA 3412 sur les apprentissages réalisés par les internautes des forums de santé en ligne. L'objectif est d'identifier les circonstances, les conditions d'utilisation de ces forums de santé ainsi que de caractériser l'apprentissage qui s'y déroule. Cela permettrait d'orienter les programmes d'ETP existants en tenant compte de ce que les internautes patients peuvent apprendre au moyen du numérique. Elle travaille également sur la modélisation de l'éducation thérapeutique du patient pluripathologique.

Rémi Gagnayre. Rémi Gagnayre est Professeur des Sciences de l'Education et de la formation, médecin. Il dirige le Laboratoire Educations et Pratiques de santé EA 3412. Ses travaux portent sur le pouvoir de décider et d'agir des personnes concernant leur santé au moyen des éducations en santé et de l'accompagnement. Il s'intéresse particulièrement à deux formes d'éducation : l'éducation thérapeutique et l'éducation à la santé des familles et leur contribution à soutenir l'acquisition de compétences en santé élément clés du pouvoir d'agir. Il est actuellement Vice Président scientifique de la Société d'Education thérapeutique Européenne. 